\title{
El efecto diferencial de escribir sobre un mismo trauma o sobre diferentes traumas en el bienestar psicológico y el estado de salud autoinformado*
}

\author{
The Differential Effect of Writing about a Same Trauma \\ or about Different Traumas on Both the Psychological \\ Well-Being and the Self-Reported Health State
}

Recibido: octubre 7 de 2009 | Revisado: marzo 28 de 2010 | Aceptado: abril 14 de 20102009

\author{
Wenceslao Peñate ** \\ TASMANia del Pino-Sedeño \\ JUAN MANUEl BETHENCOURT \\ Facultad de Psicología, Universidad de La Laguna, \\ Tenerife, Islas Canarias, España
}

Para citar este artículo. Peñate, W., Del Pino-Sedeño, T. \& Bethencourt, J.M. (2010). El efecto diferencial de escribir sobre un mismo trauma o sobre diferentes traumas en el bienestar psicológico y el estado de salud autoinformado. Universitas Psychologica, 9 (2), 433-445.

Artículo de investigación experimental. Esta investigación ha sido financiada por la Agencia Canaria de Investigación, Innovación y Sociedad de la Información (SolSubC200801000084), y por el Ministerio de Ciencia e Innovación de España PSI2009-09836

** Grupo de investigación de la Universidad de La Laguna: Abordaje diferencial de los constructos de ansiedad y depresión. Dto. Personalidad, Evaluación y Tratamientos Psicológicos, Facultad de Psicología, Universidad de La Laguna, 38204 La Laguna, Tenerife, Islas Canarias. Correos electrónicos: wpenate@ ull.es
RESUMEN

El presente trabajo se inscribe dentro de los estudios sobre la eficacia de la reexperimentación emocional de eventos traumáticos. 39 mujeres estudiantes fueron asignadas a tres condiciones experimentales: escribir sobre un mismo trauma, escribir sobre un trauma diferente y escribir sobre cuestiones triviales. Como variables dependientes se tomaron el afecto positivo y negativo, el nivel de síntomas de estrés postraumático, el nivel de depresión y el nivel de salud. A los dos meses se observó una disminución de la sintomatología de estrés postraumático y de la salud física para el grupo que escribió sobre el mismo trauma. El nivel de depresión también disminuyó, tanto para el grupo que escribió sobre el mismo trauma como para el que escribió sobre traumas distintos. A pesar de que el modelo de la exposición cognitiva es el que consigue un mayor apoyo, los resultados se discuten de acuerdo a nuevos modelos explicativos más comprehensivos.

Palabras clave autores

Reexperimentación emocional, instrucciones de escritura, salud física y bienestar psicológico.

Palabras clave descriptores

Adaptación (psicología), actitud frente a la salud, depresión mental.

\section{A B S T R A C T}

The present article is inserted into the studies on the effectiveness of the emotional disclosure of traumatic events. 39 women students were assigned to three experimental conditions: to write on a same trauma, to write about different traumas, and to write on trivial affaires. As dependent variables, the positive and negative affect, the level of symptoms of post-traumatic stress disorder, the level of depression and the level of health, were assessed. Two months later, a significant diminution of both symptoms of post-traumatic stress and symptoms of physical health for the group that wrote on the same trauma was observed. The depression level also diminished, both for the group that wrote on the same trauma, and for that wrote on different traumas. Although the model of the cognitive exposure has got a greater support, the results are discussed according to the need for a new and more extended explanatory model.

Key words authors

Emotional Disclosure, Instructions of Writing, Physical Health and Psychological Well-Being.

Key words plus

Adjustment (Psychology), Attitude to Health, Depression, Mental. 
La vivencia de traumas o situaciones altamente estresantes es relativamente frecuente. Experiencias como accidentes, catástrofes, abusos y violencia están presentes en la vida de muchas personas. Tal y como se indica en una revisión reciente (Tennant, 2006), esas vivencias no son inocuas, generando dificultades serias en el ajuste psicológico que, cuando esas experiencias pasan a formar parte de la vida cotidiana (como ocurre en algunos ambientes que nos caen muy cercanos), la probabilidad de sufrir un trastorno psicológico es muy elevada (Alejo, Rueda, Ortega \& Orozco, 2007).

A finales del siglo pasado, James Pennebaker propuso un nuevo paradigma de investigación, sobre los beneficios que producía el escribir sobre esas experiencias traumáticas, estresantes o altamente molestas. Este procedimiento se ha conocido como autorevelación emocional, apertura emocional, revelación experimental o reexperimentación emocional. El resultado ha sido muy fructífero, demostrando su utilidad en muchos y variados indicadores de salud. Así, la investigación iniciada por Pennebaker y colaboradores ha puesto en evidencia que el procedimiento de reexperimentación emocional (RE), se relaciona con una mejora en la salud física (Pennebaker \& Beall, 1986; Pennebaker, Colder \& Sharp, 1990; Pennebaker \& Francis, 1996), mejor funcionamiento físico (Pennebaker et al., 1990; Spera, Buhrfeind \& Pennebaker, 1994); mejor manejo de los procesos de enfermar, incluyendo la fase terminal (Cepeda et al., 2008; D'Souza, Lumley, Kraft \& Dooley, 2008; Gillis, Lumley, Mosley-Williams, Leisen \& Roehrs, 2006; Keefe et al., 2008; Warner et al., 2006) mejora de la función inmune y marcadores biológicos (Esterling, Antoni, Fletcher, Margulies \& Schneiderman, 1994; Pennebaker, Hughes \& O'Heeron, 1987; Pennebaker, Kiecolt-Glaser \& Glaser, 1988; Petrie, Booth, Pennebaker, Davison \& Thomas 1995; Petrie, Fontanilla, Thomas, Booth \& Pennebaker, 2004). Asimismo, el procedimiento ha mostrado su eficacia en la disminución significativa en síntomas psicológicos (Schoutrop, Lange, Hanewald, Davidovich \& Salomon, 2002; Sloan \& Marx, 2004; Sloan, Marx \& Epstein, 2005). Igualmente, se ha observado la eficacia del pro- cedimiento en personas que informaron al menos de una gravedad moderada de síntomas de estrés postraumático (Sloan \& Marx, 2004; Sloan et al., 2005). Incluso se ha encontrado su utilidad en procesos y actividades tan variadas como la disminución del absentismo laboral (Francis \& Pennebaker, 1992); o la mayor probabilidad de obtener nuevo empleo (Spera, Buhrfeind \& Pennebaker, 1994).

Mayores controversias se han encontrado con respecto a los procesos depresivos. Desde los primeros trabajos sobre la RE (Smyth, 1998), uno de los resultados más consistentes es el efecto iatrogénico que provoca inicialmente sobre el bienestar psicológico (aumentando, entre otros síntomas, el estado de ánimo deprimido y el humor negativo). Este efecto tiende a disiparse sesión a sesión. Cuando ese estado de ánimo pasa a convertirse en un proceso depresivo, usualmente vinculados a la pérdida de seres queridos, los resultados no evidencian la eficacia de la RE (Lepore, 1997; Stroebe, Schut \& Stroebe, 2006, 2005). Frente a la inutilidad de la RE para los procesos depresivos, algunos autores han señalado que puede ser eficaz en los procesos depresivos cuando se utiliza como una estrategia de ayuda dentro de un proceso psicoterapéutico más amplio (Graf, Gaudiano \& Geller, 2008). A la vista de estos resultados, se ha llegado a plantear la utilidad de la RE como herramienta terapéutica útil, sencilla, manejable y de fácil acceso (Lepore \& Smyth, 2002; McCurdy, 2001; Pennebaker, 1997).

Para explicar los motivos por los que la RE funciona, se han elaborado distintos modelos explicativos. Inicialmente Pennebaker (1989) elaboró un modelo basado en un sistema de inhibición conductual, heredero de la catarsis freudiana, según el cual la RE era eficaz en la medida en que liberaba al organismo del coste que suponía la inhibición de los traumas o vivencias altamente estresantes. Posteriormente observó que la desinhibición no era una razón suficiente para explicar la eficiencia de la $\mathrm{RE}$, por lo que añadió el procesamiento cognitivo del trauma, en la medida en que su evocación permitía un mejor conocimiento y asimilación (Pennebaker, 1993). Posteriormente, se han desarrollado otros modelos explicativos. Para nuestro interés, cabe destacar la propuesta de Bootzin (1997), por la 
que la RE funcionaría como una suerte de exposición cognitiva, al modo de la técnica de exposición, generando, con la exposición repetida, un proceso de habituación.

A partir de los trabajos iniciales, el desarrollo experimental y las distintas aplicaciones de la RE han sido muy importantes. Metodológicamente, se han llevado a cabo tres meta-análisis sobre su eficacia, las razones para esa eficacia y para quiénes es eficaz. Smyth, en 1998 revisó 13 artículos. Posteriormente, Frisina, Borod y Lepore (2004), revisaron 9 estudios más (sólo uno era compartido con la revisión anterior); y, por último, Frattaroli (2006), revisó, con unos criterios más amplios, 146 trabajos. Los tres análisis concuerdan en que la RE posee efectos beneficiosos (aunque varía el tamaño del efecto, desde 0,075 a 0,23). Tomando como referencia el último meta-análisis de Frattaroli (2006), los resultados indican que la RE es útil en personas con problemas físicos y mentales (especialmente en el estrés postraumático). Con respecto a las condiciones experimentales y el diseño, la RE ha mostrado ser más eficaz cuando la apertura emocional se hace en privado, sobre problemas recientes, se les instruye específicamente sobre qué reexperimentar y sobre si cambiar o no de tema, se llevan a cabo al menos tres sesiones, con una duración cada una de 15 minutos o más ${ }^{1}$, y se hace un seguimiento de un mes o menos. Otras variables que no han podido mostrar su participación en la eficacia de la RE, son los criterios de selección por problemas de salud, la edad, el nivel educativo de los participantes, la valencia de los temas seleccionados para reexperimentar, o el modo de reexperimentar (escritura, hablar).

Como puede observarse, todavía quedan muchas lagunas por resolver. En nuestro caso, nos planteamos el papel de las instrucciones experimentales de la revelación, especialmente debido a la llamada de atención sobre un uso no adecuado de esas instrucciones de acuerdo con el paradigma de

1 A pesar de la consistencia de estos hallazgos en relación con el número de sesiones y su duración, todavía aparecen resultados que hablan a favor de formatos más cortos (dos minutos) y menos sesiones (2 sesiones) (Burton \& King, 2008).
Pennebaker (Batten, Follette, Hall \& Palm, 2002; Sloan, Marx, Epstein \& Lexington, 2007). Sloan et al. (2005) han sugerido que aunque las instrucciones estándar para la tarea de reexperimentación permiten al individuo una escritura sobre diferentes temas en cada sesión, la ganancia terapéutica puede ser obtenida a través de la escritura de la misma experiencia, más que por la escritura de distintas experiencias en cada sesión. Así, algunos autores consideran el paradigma de RE como un contexto que permite al individuo exponerse al estímulo negativo condicionado (v.g., los recuerdos) у а otros estímulos que han sido previamente evitados (Bootzin, 1997; Kloss \& Lisman, 2002; Lepore, Greenberg, Bruno \& Smyth, 2002; Pennebaker, 1997). Con lo que la efectividad del paradigma de RE estaría en función de la exposición repetida del mismo trauma a lo largo de las sesiones.

En este sentido, específicamente, nos planteamos si supone lo mismo indicar a los participantes que reexperimenten todo el tiempo un mismo trauma o que elijan un nuevo trauma para cada sesión de RE. Teóricamente, si el modelo de inhibición conductual es el que explica el funcionamiento de la RE, daría igual si se reexperimenta un mismo trauma que uno diferente cada vez. En cambio, si es el modelo de exposición cognitiva, lo que realmente sería eficaz, es la exposición repetida a un mismo trauma. Secundariamente nos interesó conocer si el tiempo de la sesión podía modular los resultados. Partiendo de los datos que indican que la RE es eficaz a partir de los 15 minutos de sesión, nos planteamos si es igual de eficaz 15 minutos que el doble de tiempo (30 minutos).

\section{Método}

\section{Participantes}

La muestra final que completaron todas las sesiones de reexperimentación emocional y el seguimiento estuvo compuesta por 39 estudiantes de tercer año de la Licenciatura de Psicología de la Universidad de La Laguna. El total de los participantes eran mujeres y la media de edad de los participantes fue de 21,8 años, el 70\% tenía 20 o 21 años (rango 
de 20 a 30 años). La participación fue voluntaria, pudiendo abandonar el experimento cuándo deseasen. Como contrapartida por su participación, recibieron créditos en una de las materias de los estudios.

\section{Instrumentos}

A continuación, se presentan los instrumentos de medida que se adoptaron para evaluar los posibles cambios tras la administración del procedimiento de reexperimentación emocional y su monitorización.

\section{Escala Diagnóstica de Estrés Postraumático (PDS)}

La PDS (Foa, 1996) es una medida de autoinforme de 17 ítems diseñada para ayudar en la detección y el diagnóstico de trastorno por estrés postraumático (TEPT), estrechamente relacionados con los criterios recogidos en el DSM-IV (APA, 1994). Se ha incluido su uso para monitorizar los efectos de la RE sobre los síntomas del TEPT. La escala se responde de acuerdo a los síntomas que han experimentado en el último mes. La PDS ha demostrado ser un instrumento psicométricamente sólido (Foa, Cashman, Jaycox \& Perry, 1997).

\section{Cuestionario de Sucesos Vitales (CAPS)}

Se administró una de las escalas de la CAPS (Blake et al., 1995) de 17 items (cuestionario de sucesos vitales, CSV), en la que se describen una serie de acontecimientos difíciles o angustiosos a los que el ser humano puede estar expuesto. Los participantes deben marcar si les ha ocurrido, si fueron testigos, si se enteraron de ello, si no están seguros o si no se aplican estos sucesos a lo largo de toda su vida. Entre los sucesos vitales estresantes o angustiosos que se miden, se encuentran los desastres naturales, fuego o explosión, accidentes con medios de transporte, exposición a sustancias tóxicas, ataques físicos, ataques sexuales, enfermedad o lesiones que amenazan la vida; muertes violentas y/o repentina de alguien próximo, etc. Tanto la versión original como la versión en castellano han mostrado buenos ajustes psicométricos (Orengo, Aburto \& Ormaechea, 2000). Este inventario se utilizó con la finalidad de sensibilizar y facilitar el recuerdo de hechos estresantes, dolorosos o traumáticos.

La Escala de Ansiedad y Depresión Hospitalaria (HADS)

Está formada por 14 ítems distribuidos en dos subescalas, siete de ansiedad y siete de depresión (Zigmond \& Snaith, 1983). Se centra en los aspectos emocionales y cognitivos, eliminándose la sintomatología somática de esos dos trastornos. Se responde de acuerdo a cómo se ha sentido en la última semana y permite valorar la presencia de ansiedad o depresión en tres niveles: si los valores son inferiores a 8 , ausencia; entre 8 y 10 , posible o dudosa; y probable o afirmativa si son de 11 o superiores. La escala posee buenos ajustes psicométricos, incluyendo en la versión española (Quintana et al., 2003).

Escala de sintomas físicos

Es un autoinforme desarrollado por los autores del presente estudio, para medir la presencia y frecuencia durante el último mes (en una escala de $\mathrm{O}$ a 3 ) de determinadas molestias físicas, donde se presuma una disminución de la eficacia del sistema inmunológico (problemas de las vías respiratorias, infecciones en la cavidad bucal, infecciones cutáneas...). Con las respuestas se obtiene un sumatorio a modo de índice de salud/enfermedad física, donde a mayor puntuación, peor salud. Finalmente, se solicita que informen sobre las visitas médicas que han realizado (eliminando las visitas programadas o por enfermedades crónicas). En el anexo se recoge una copia de esta escala.

Escala de afecto positivo y negativo (PANAS)

Esta escala (Watson, Clark \& Tellegen, 1988) contiene 20 descriptores de estados de ánimo (10 positivos y 10 negativo). La validación psicométrica (Watson et al., 1988) ha mostrado la existencia 
de una estructura bifactorial de afecto positivo (AP) y afecto negativo (AN), que funcionan como dos subescalas independientes. Esta estructura bifactorial también se ha verificado en la versión española (Sandín et al., 1999). En este estudio la PANAS se ha utilizado para monitorizar el estado de ánimo sesión a sesión²

\section{Diseño}

Se realizó un diseño multigrupo con una variable independiente principal que consistía en el número de traumas ${ }^{3}$ (con dos niveles: contar un mismo trauma o contar distintos traumas a lo largo de las sesiones). Secundariamente se introdujo una segunda variable independiente, tiempo de la sesión (con dos niveles: 15 minutos o 30 minutos de escritura), para observar si la duración de la sesión participaba de los efectos de la RE. Asimismo se contó con un grupo control que escribió sobre cuestiones triviales en sesiones de 30 minutos. Se tomaron como variables dependientes el nivel de síntomas de estrés postraumático, el nivel de ansiedad y de depresión, el nivel de salud, y el afecto positivo y negativo. Los participantes fueron asignados aleatoriamente a las cinco condiciones experimentales (escribir sobre el mismo trauma durante 15 minutos, escribir sobre traumas distintos durante 15 minutos, escribir sobre el mismo trauma durante 30 minutos, escribir sobre traumas distintos durante 30 minutos, y escribir sobre cuestiones triviales durante 30 minutos). Las sesiones de escritura fueron tres, realizadas en días consecutivos.

\section{Procedimiento}

Los participantes fueron informados de que tendrían que escribir sobre una situación personal con el fin de entender cómo las personas reaccionan a esas situaciones. Se les informó de que sus cuestionarios y materiales serían guardados confidencial-

2 De acuerdo con el modelo tripartito de Clark y Watson (1991), el NA estaría compartido por la ansiedad y la depresión, y el bajo PA (anhedonia) sería prototípico de la depresión.

3 Por 'trauma' se va a entender la vivencia de un hecho estresante, altamente molesto/doloroso o traumático. mente, y así se hizo constar en el formulario de consentimiento informado, que fue firmado por todos. Los participantes fueron asignados aleatoriamente a la condición de contar un trauma, contar varios traumas o a la condición de situación trivial hasta completar 20 participantes para cada uno de ellas. A su vez estos se dividían en 10 para 15 minutos de redacción y 10 para 30 minutos (el grupo control redactó siempre durante 30 minutos).

Las instrucciones dadas a los participantes de trauma fueron una modificación de las instrucciones de Pennebaker et al. (1988), para añadir así las variables tiempo (15 o 30 minutos) y número de traumas (un trauma, distintos trauma y situaciones triviales).

Por ejemplo, a los participantes asignados a la condición de un mismo trauma durante 30 minutos, se les dio las siguientes instrucciones:

Primer día: Los seres humanos estamos expuesto a una serie de hechos que no podemos controlar y que nos suponen un cierto daño psicológico (accidentes de tráfico, desastres naturales, muertes de seres queridos, observar la muerte o el suicidio de alguien, ser asaltado, sufrir abusos sexuales, ser violado...). Ahora trata de recordar un hecho que te haya ocurrido o que hubieras presenciado y que te haya resultado altamente molesto, muy estresante o traumático. Preferiblemente céntrate en el que te haya parecido más traumático. Concéntrate en todos los detalles (sonidos, olores, personas, lugares, colores...) cierra los ojos si ello te ayuda a concentrarte. Haz pasar esa historia por tu mente y, a continuación, descríbela durante 30 minutos, escribiendo todos los detalles que recuerdes, acompañado de tus sensaciones y tus emociones. Escribe desde lo que sucedió antes del hecho hasta después de pasado el mismo. No te preocupes por la ortografía, la sintaxis o la gramática, solo es interesante que describas tus sentimientos y emociones más profundas asociada a esos hechos. Segundo día: Vuelve a concentrarte en la situación traumática, estresante o altamente molesta anterior. De nuevo, concéntrate en todos los detalles (sonidos, olores, personas, lugares, colores...) y, a continuación, descríbela durante 30 minutos, escribiendo todos los detalles que recuerdes, acompañado de tus sensaciones y tus emociones. Escribe desde lo que 
sucedió antes del hecho hasta después de pasado el mismo. No te preocupes por la ortografía, la sintaxis o la gramática. Recuerda que lo importante es que describas tus sentimientos y emociones más profundas asociada a esos hechos en el orden en que ocurrieron. Tercer día: Por última vez, vuelve a concentrarte en la situación traumática, estresante o altamente molesta anterior. De nuevo, concéntrate en todos los detalles (sonidos, olores, personas, lugares, colores...) y, a continuación, descríbela durante 30 minutos, escribiendo todos los detalles que recuerdes, acompañado de tus sensaciones y tus emociones. Escribe desde lo que sucedió antes del hecho hasta después de pasado el mismo. No te preocupes por la ortografía, la sintaxis o la gramática. Recuerda que lo importante es que describas tus sentimientos y emociones más profundas asociada a esos hechos en el orden en que ocurrieron.

A los participantes asignados a la condición de situaciones triviales les fue dada instrucciones como las siguientes:

Durante 30 minutos, trata de recordar cronológicamente todo lo que has hecho hoy, desde que te levantaste hasta que llegaste aquí. Concéntrate y haz pasar el desarrollo de este día por tu mente y, a continuación, escríbelo, describiendo todo los detalles que recuerdes, acompañados de tus sensaciones y tus emociones. No te preocupes por la ortografía, la sintaxis o la gramática.

A todos los participantes se les comunicó que sus datos serían confidenciales y que sólo podrían acceder a ellos los investigadores de este estudio, por lo que podían responder con total sinceridad y tranquilidad a todas las preguntas.

Según iban llegando los participantes, se les informaba individualmente del objetivo del estudio y se les permitía preguntar cualquier duda. Tras esto, firmaban el formulario de consentimiento informado y se les suministró el dossier de pruebas (CSV, PDS, HAD y escala de salud) y las instrucciones adecuadas a la condición experimental a la que se le asignaba. A continuación, completaban la primera sesión de escritura. Inmediatamente después cumplimentaban la escala PANAS. Dos meses después se les volvía a administrar los mismos cuestionarios e inventarios (salvo el CSV).

Los participantes obtuvieron créditos como reconocimiento por su colaboración en una asignatura del grado de Psicología.

\section{Resultados}

Un primer análisis se llevó a cabo para reconocer el nivel de abandonos (personas que no completaron todas las fases, hasta el seguimiento). En la Tabla 1 se resumen los datos:

TABLA 1

Participantes en las condiciones de tiempo de redacción (15 y 30 minutos), de tipo de trauma (escribir sobre el mismo o sobre distintos traumas, sesión a sesión) y control

\begin{tabular}{lcccc}
\hline & \multicolumn{3}{c}{ Grupo } \\
\cline { 3 - 5 } & & $\begin{array}{c}\text { Mismo } \\
\text { trauma }\end{array}$ & $\begin{array}{c}\text { Varios } \\
\text { traumas }\end{array}$ & Control \\
\hline \multirow{2}{*}{ Tiempo } & 15 minutos & 9 & 6 & 10 \\
& 30 minutos & 9 & 5 & \\
Total & 18 & 11 & 10 \\
\% abandonos sobre 20 & 10 & 45 & 50 \\
\hline
\end{tabular}

Fuente: elaboración propia.

Como puede observarse, la tasa de abandonos es muy desigual, destacando la adherencia de las personas que redactaron los tres días el mismo trauma, mientras que la tasa de abandonos fue elevada, tanto para la condición de contar diferentes traumas cada día, como la condición de control.

Entrando a comentar los resultados obtenidos, en primer lugar se quiso conocer los efectos contextuales, sesión a sesión y seguimiento, del protocolo de RE llevado a cabo. Para ello se realizó un análisis de varianza de medidas repetidas (MANOVA) sobre los dos factores del PANAS. Con respecto al AN, se observó una disminución estadísticamente significativa, $F=5,26(p \leq 0,03)$, con un tamaño del efecto apreciable $\left(\eta^{2}=0,226\right)$. Este resultado fue producto del efecto sesión a sesión, sin que se observaran otros efectos de otras 
variables (mismo o diferente trauma, escribir $15 \mathrm{o}$ 30 minutos). Con respecto al afecto positivo, los resultados son similares, salvo que en este caso se produce un aumento significativo del AP sesión a sesión $F=6,519\left(p \leq 0,02 ; \eta^{2}=0,227\right)$. En la Figura 1 se puede observar esa evolución.

\section{FIGURA 1}

Nivel de afecto negativo y de afecto positivo en las tres sesiones de escritura y en el seguimiento medidos por el PANAS. $\mathrm{N}=39$.

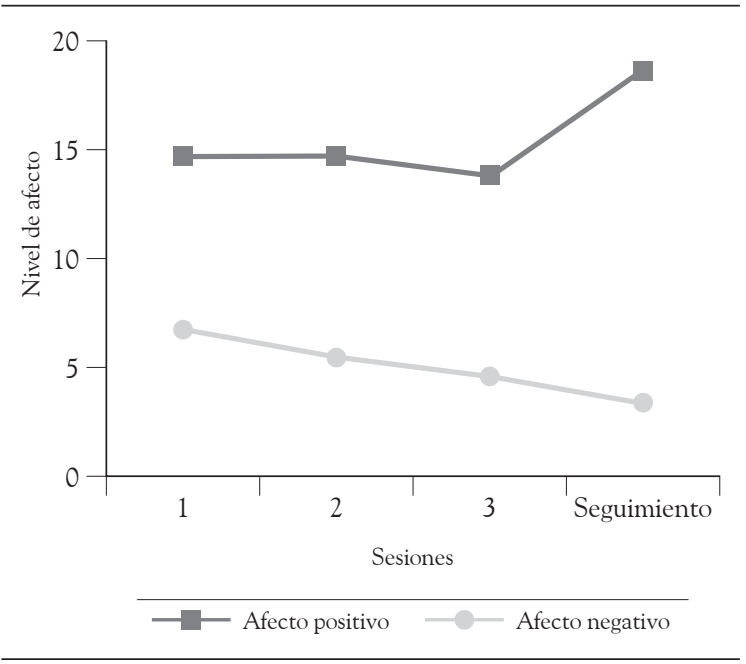

Fuente: elaboración propia.

Con respecto a los efectos no contextuales, se realizaron de nuevo análisis de varianza con medidas repetidas, tomando las medidas previas y las medidas del seguimiento para los niveles de ansiedad y depresión (HADS), síntomas de estrés postraumático (PDS) y nivel de salud (escala de síntomas físicos). Así, en relación con la ansiedad, no se observaron efectos significativos algunos. Sin embargo, para el nivel de depresión se encontraron diferencias significativas en las medidas intersujetos entre los grupos en función del tiempo de redacción, $F=7,43\left(p \leq 0,01 ; \eta^{2}=0,271\right)$. Tal y como puede observarse en la en la Figura 2, parece que el grupo de narración de un trauma durante 30 minutos disminuye su nivel de depresión, mientras que el grupo control y el de narración corta muestran peores índices post RE.

Para la sintomatología TEPT, se observó una variación significativa en la puntuación total en la PDS en función del número de traumas expuestos, $F=6,05(p \leq 0,01)$, con un tamaño del efecto apreciable $\left(\eta^{2}=0,482\right)$. El grupo de narración de un mismo trauma disminuye más sus puntuaciones en comparación con el grupo control y el grupo que narró diferentes traumas (que incluso aumentan sus puntuaciones). En la Figura 3 se puede observar ese efecto.

\section{FIGURA 2}

Nivel de depresión (pre y seguimiento) para el tiempo de redacción (15 o 30 minutos) medido por la HADS.

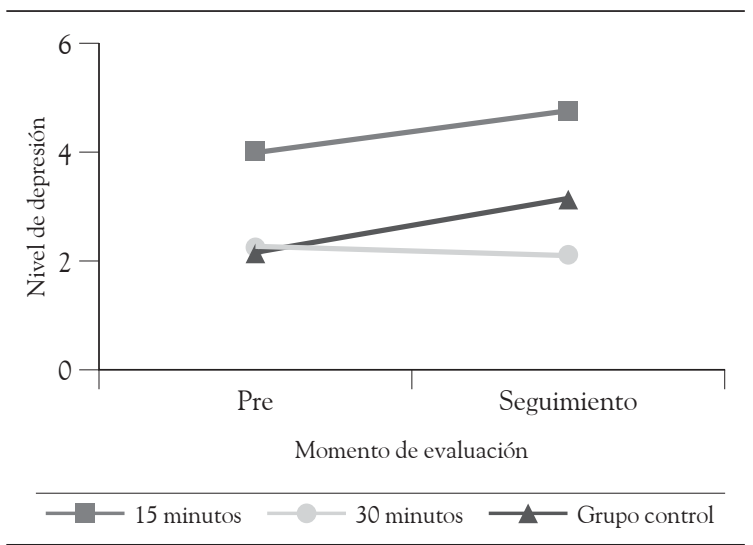

Fuente: elaboración propia.

\section{FIGURA 3}

Síntomas de estrés postraumático (pre y seguimiento) según el tipo de trauma sobre el que se escribe (sobre el mismo, variar sesión a sesión), medidos por el PDS.

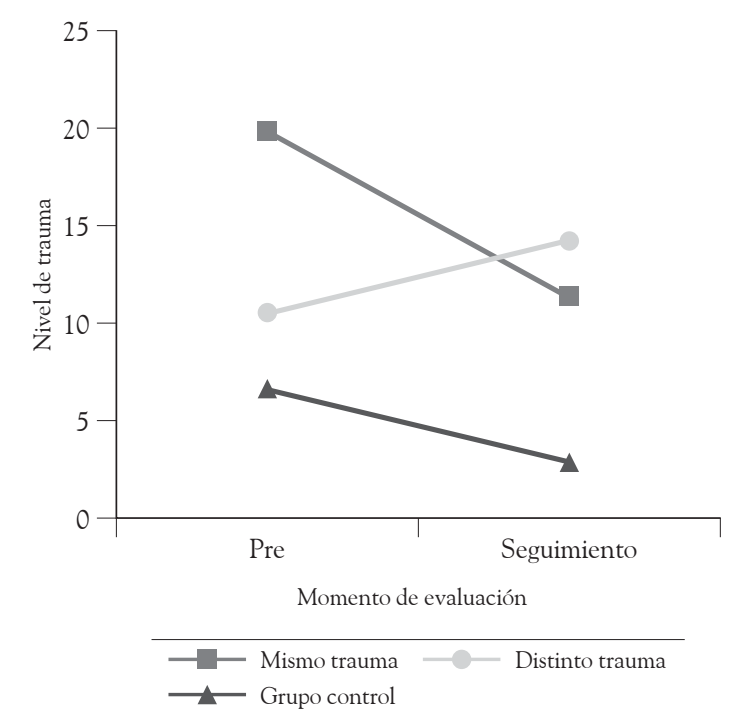

Fuente: elaboración propia. 
Por último, para el índice de salud se obtuvo un efecto significativo pre-seguimiento en la interacción del grupo por tiempo, $F=4,31(p \leq 0,05$; $\left.\eta^{2}=0,17\right)$ : el grupo de narración de un mismo trauma durante 30 minutos mejoró su estado de salud (se han invertido las puntuaciones para indicar que a mayor puntuación mejor salud), mientras que el resto de los grupos tuvieron peores resultados en el seguimiento. El grupo con peor evolución fue el grupo de narración de distintos traumas durante 30 minutos, mientras que los grupos de 15 minutos tuvieron una pequeña caída en salud, similar a la del grupo control (Figura 4).

En resumen, se ha observado determinados efectos de la RE en medidas como afecto positivo y negativo, depresión, síntomas de TEPT y salud, especialmente en relación con contar siempre el mismo trauma (y, secundariamente, con hacerlo durante 30 minutos).

\section{Discusión}

Este estudio examinó cómo el paradigma de RE, que se lleva a cabo a través de la escritura de situaciones traumáticas, altamente molestas o estresantes, se relaciona con salud física y psicológica.
Específicamente, se han explorado los beneficios de la escritura repetida cuando se realiza sobre un mismo trauma o sobre distintos traumas, durante tres sesiones de escritura. Secundariamente, se observó si se obtenían los mismos beneficios con una escritura corta, de 15 minutos, o con una escritura más de más tiempo: 30 minutos.

En primer lugar, se pudo constatar como la $\mathrm{RE}$ posee un cierto efecto iatrogénico inmediato, sobre el estado de ánimo más situacional (en este caso sobre la afectividad positiva y negativa), que se va disipando sesión a sesión, hasta el punto que en el seguimiento llega a alcanzar los niveles más adaptativos (menor afectividad negativa y mayor afectividad positiva). Hay que señalar que estos efectos fueron independientes de escribir sobre un mismo o diferente trauma.

Con respecto a los resultados en relación con los marcadores de salud física y mental, a grandes rasgos, parece que los mayores beneficios se obtienen cuando se escribe sobre un mismo trauma. Así, se han podido verificar mejoras en los niveles de depresión, en la sintomatología de estrés postraumático y sobre la salud física en general. Frente a la propuesta inicial de Pennebaker (Pennebaker \& Beall, 1986; Pennebaker et al., 1990; Pennebaker

Figura 4

Nivel de salud autoinformado (pre y seguimiento) para el tiempo de redacción (15 030 minutos) y el tipo de trauma (escribir sobre el mismo, variar sesión a sesión), medido por la escala de salud física.

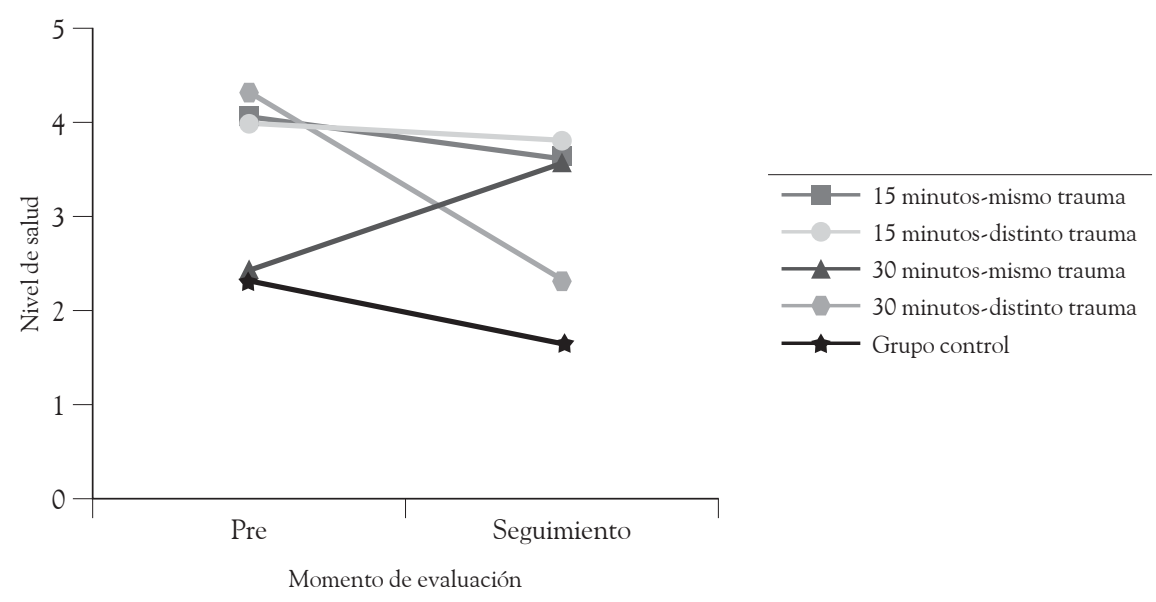

Fuente: elaboración propia. 
\& Francis, 1996), no ofrece el mismo resultado el escribir sobre un mismo trauma que sobre diferentes traumas. En este sentido, los resultados obtenidos van más en la línea de Sloan, Marx y Epstein (2005), que encontraron que lo realmente beneficioso consistía en escribir repetidamente sobre el mismo hecho. De esta forma, parece favorecerse una interpretación de la eficacia de la RE en relación con un proceso de habituación, más que a la alteración de un mecanismo de inhibición conductual.

De hecho, los resultados son algo paradójicos en relación con la RE y la salud física: lo que parece ocurrir no es tanto que mejoren las personas que escriben sobre un mismo hecho (que mejoran), sino que las personas que escriben sobre distintos hechos empeoran o permanecen estables. Realmente, hay un dato que favorece aún más la hipótesis de la habituación y es que la RE es efectiva cuando más tiempo se está escribiendo (30 minutos), ya que los que escribieron sobre un mismo trauma durante 15 minutos empeoraron ligeramente.

Los datos obtenidos en relación con los niveles de sintomatología TEPT permiten observar más claramente ese efecto paradójico: los participantes que narraban el mismo trauma disminuyeron su sintomatología frente al grupo control, mientras que el grupo de narración de distintos traumas aumentó su sintomatología (como si escribir sobre un trauma en una única ocasión les sensibilizara). En todo caso, los resultados van en la línea de la eficacia de la RE en personas que informaron al menos de una gravedad moderada de síntomas de estrés postraumático (Sloan \& Marx, 2004; Sloan et al., 2005).

Estos dos resultados (sobre la salud física y sobre la sintomatología TEPT), apoyan las tesis defendidas por algunos autores que afirman que el paradigma de escritura sirve como un contexto que permite al individuo exponerse al estímulo negativo: a través de la exposición repetida a esos estímulos, la escritura expresiva extingue la respuesta emocional negativa asociada (Bootzin, 1997; Kloss \& Lisman, 2002; Lepore et al., 2002). Por otro lado, contradicen las propuestas del propio Pennebaker (1997) sobre la mayor solidez de la RE, cuando los participantes pueden elegir el trauma en cada sesión, o cuando se les instruye para que escriban sobre temas específicos de trauma (Batten et al., 2002). Queda por establecer los datos aportados por el meta-análisis llevado a cabo por Smyth (1998) sobre la menor eficacia cuando se instruye para que los participantes redacten sobre traumas del pasado. En todo caso, el hecho de que parte de la efectividad del paradigma se encuentre en la libre elección del trauma, no contradice el hecho de que la escritura repetida de un mismo trauma, una vez realizada la elección, pueda proveer de cierta eficacia el procedimiento.

En cuanto al nivel de depresión, la eficacia de la RE sí parece independiente de si se escribe sobre el mismo o diferente trauma. En este caso, el dato más relevante está en el tiempo de escritura: el nivel de depresión disminuye cuando se escribe durante más tiempo (30 minutos). En este sentido, este dato está estrechamente relacionado con los resultados ya citados sobre el AN y el AP (que a su vez son afectos básicos vinculados a los procesos depresivos). En este caso, frente a lo que ocurría con la salud física (y su posible vinculación con el sistema inmune) y con los síntomas directamente vinculados al trauma, la depresión parece más vinculada con un elemento metacognitivo: el recuperar un hecho doloroso desestructura emocionalmente (con independencia del hecho traumático recordado), y sólo la exposición prolongada logra disminuir esa desestructuración. De alguna manera, la depresión sería una especie de atributo del hecho de recordar un suceso doloroso, sin relación con la naturaleza o repetitividad del recuerdo: lo decisivo para que la RE reduzca los niveles de depresión consistiría en exponerse al desánimo en sí mismo durante mayor tiempo, sin relación con el suceso que lo evoque. En este caso la hipótesis de la exposición no parece tan sólida y sí parece favorecer la hipótesis de la existencia de un mecanismo de inhibición conductual alterado, como ha defendido James Pennebaker.

El hecho de que se planteen hipótesis explicativas alternativas en función de la naturaleza de la variable dependiente tomada en consideración, complica aún más la justificación del fun- 
cionamiento de la RE. Evidentemente, se podrían aceptar mecanismos duales de funcionamiento, pero también se podrían observar nuevas hipótesis explicativas nuevas. En este sentido, los planteamientos derivados de la teoría del marco relacional y, más específicamente, de la terapia de aceptación y compromiso (Hayes, Barnes-Holmes \& Roche, 2001; Wilson \& Luciano, 2002), postulando que la evitación es la clave del sufrimiento humano, podría justificar por qué la exposición repetida a un hecho traumático evitado logra mejorar el ajuste físico y psicológico, sin relación con el hecho traumático en sí y si se expone al mismo o distinto suceso. En todo caso es una nueva hipótesis que necesitaría ser contrastada.

Lo comentado hasta aquí se deriva de un trabajo con claras limitaciones: se ha trabajado con una muestra de estudiantes mujeres, con participación voluntaria, sin conocer su historial de TEPT y las implicaciones que pudiera tener el hecho recordado para su ajuste personal, o el papel mediador de otras variables (como la alexitimia o el optimismo). Además, algunas medidas de autoinforme (especialmente el nivel de salud/enfermedad) necesitarían de un soporte más objetivado, y también se necesitaría de un seguimiento mayor. Con todo, la limitación más importante está en el tamaño de la muestra, producto especialmente de los abandonos. En este sentido, reconociendo sus limitaciones, resulta relevante destacar el nivel de abandonos como una aportación positiva: la mayor adherencia se produjo en la muestra que escribió sobre un mismo hecho. Este dato puede ser interesante desde un planteamiento de la RE como recurso terapéutico. En todo caso, la promoción de la escritura como abordaje psicológico único parece prematuro, ya que dicha práctica no ha sido justificada por los resultados empíricos (Batten et al., 2002) y se disponen de otras alternativas psicoterapéuticas plausibles para el daño psicológico de la vivencia de traumas (v.g., Botero-García, 2005). Los resultados del presente estudio, junto con los de investigaciones anteriores sugieren que, en el mejor de los casos, su uso debe limitarse a servir como un complemento a otros tratamientos apoyados empíricamente (Sloan \& Marx, 2006).
En conclusión, la RE ha mostrado su capacidad para disminuir los niveles de síntomas de TEPT, de depresión y una mejora en el nivel de salud autopercibido. Esta mejora está en relación con escribir sobre un mismo trauma y con escribir durante un periodo de tiempo más prolongado.

\section{Referencias}

Alejo, E., Rueda, G., Ortega, M. \& Orozco, L. C. (2007). Estudio epidemiológico del trastorno por estrés postraumático en población desplazada por la violencia política en Colombia. Universitas Psychologica, 6, 623-635.

American Psychiatric Association. (1994). Diagnostic and Statistical Manual of Mental Disorders. DSMIV. Washington D.C.: Autor.

Batten, S. V., Follette, V. M., Hall, M. L. R. \& Palm, K. M. (2002). Physical and psychological effects of written disclosure among sexual abuse survivors. Behavior Therapy, 33, 107-122.

Blake, D. D., Weathers, F. W., Nagy, L. M., Kaloupek, D. G., Gusman, F. D., Charney, D. S. \& Keane, T. M. (1995). The development of a ClinicianAdministered PTSD Scale. Journal of Trauma Stress, 8, 75-90.

Bootzin, R. R. (1997). Examining the theory and clinical utility of writing about emotional experiences. Psychological Science, 8, 167-169.

Botero-García, C. (2005). Efectividad de una intervención cognitivo-conductual para el trastorno por estrés postraumático en excombatientes colombianos. Universitas Psychologica, 4, 205-219.

Burton, C. M. \& King, L. A. (2008). Effects of (very) brief writing on health: The two-minute miracle. British Journal of Health Psychology, 13, 9-14.

Cepeda, M. S., Chapman, C. R., Miranda, N., Sánchez, R., Rodríguez, C. H., Restrepo et al. (2008). Emotional disclosure through patient narrative may improve pain and well-being: Results of a randomized controlled trial in patients with cancer pain. Journal of Pain and Symptom Management, 35, 623-631.

Clark, L. A. \& Watson, D. (1991). Tripartite Model of Anxiety and Depression: Psychometrics evidence 
and taxonomic implications. Journal of Abnormal Psychology, 3, 316-336.

D'Souza, P. J., Lumley, M. A., Kraft, C. A. \& Dooley, J. A. (2008). Relaxation training and written emotional disclosure for tension or migraine headaches: A randomized, controlled trial. Annals of Behavioral Medicine, 36, 21-32.

Esterling, B. A., Antoni, M. H., Fletcher, M. A., Margulies, S. \& Schneiderman, N. (1994). Emotional disclosure through writing or speaking modulates latent Epstein-Barr virus antibody titers. Journal of Consulting and Clinical Psychology, 62, 130-140.

Foa, E. B., Cashman. L., Jaycox. L. \& Perry, K. (1997). The validation of a self-report measure of posttraumatic stress disorder: The Posttraumatic Diagnostic Scale. Psychological Assessment, 9, 445-451.

Foa, E. B. (1996). Posttraumatic Stress Diagnostic Scale. Minneapolis, MN: National Computer Systems.

Francis, M. E., \& Pennebaker, J. W. (1992). Putting stress into words: The impact of writing on physiological, absentee, and self-reported emotional well-being measures. American Journal of Health Promotion, 6, 280-286.

Frattaroli, J. (2006). Experimental disclosure and its moderators: A meta-analysis. Psychological Bulletin, 132, 823-865.

Frisina, P. G., Borod, J. C. \& Lepore, S. J. (2004). A meta-analysis of the effects of written emotional disclosure on the health outcomes of clinical populations. Journal of Nervous $\mathcal{E}$ Mental Disease, 192, 629-634.

Gillis, M. E., Lumley, M. A., Mosley-Williams, A., Leisen, J. C. \& Roehrs, T. (2006). The health effects of at-home written emotional disclosure in fibromyalgia: A randomized trial. Annals of Behavioral Medicine, 32, 135-146.

Graf, M. C., Gaudiano, B. A. \& Geller, P. A. (2008). Written emotional disclosure: A controlled study of the benefits of expressive writing homework in outpatient psychotherapy. Psychotherapy Research, 18, 389-399.

Hayes, S., Barnes-Holmes, D. \& Roche, B. (2001). Relational frame theory: A post-skinnerian account of human language and cognition. Nueva York: Kluwer Academic/Plenum Plubishers.
Keefe, F. J., Anderson, T., Lumley, M., Caldwell, D., Stainbrook, D., McKee, D. et al. (2008). A randomized, controlled trial of emotional disclosure in rheumatoid arthritis: Can clinician assistance enhance the effects? Pain, 137, 164-172.

Kloss, J. D. \& Lisman, S. A. (2002). An exposure-based examination of the effects of written emotional disclosure. British Journal of Health Psychology, 7, 31-46.

Lepore, S.J. \& Smyth, J.M. (Eds.). (2002). The writing cure. How expressive writing promotes health and emotional well-being. Washington, DC: American Psychological Association.

Lepore, S. J. (1997). Expressive writing moderates the relation between intrusive thoughts and depressive symptoms. Journal of Personality and Social Psychology, 73, 1030-1037.

Lepore, S. J., Greenberg, M. A., Bruno, M. \& Smyth, J. M. (2002). Expressive writing and health: Selfregulation of emotion-related experience, physiology, and behavior. En S. J. Lepore \& J. M. Smyth (Eds.), The writing cure: How expressive writing promotes health and emotional well-being (pp. 99. 117). Washington, DC: American Psychological Association.

McCurdy, M. M. (2001). From trauma to writing: A theoretical model for practical use. In C. M. Anderson \& M. M. McCurdy (Eds.), Writing and healing: Toward an informed practice (pp. 158-200). Urbana, IL: National Council of Teachers of English.

Orengo, F., Aburto, M. \& Ormaechea, J. A. (2000). Valoración psiquiátrico legal de 20 personas implicadas en un accidente de tráfico con manifestaciones de estrés postraumático utilizando el Cuestionario MMPI-2 y la Escalas CAPS-DX. Psiquis, 21, 167-180.

Pennebaker, J. W. (1989). Confession, inhibition, and disease. En L. Berkowitz (Ed.). Advances in experimental social psychology (Vol. 22, pp. 211-244). San Diego, CA: Academic Press.

Pennebaker, J. W. (1997). Writing about emotional experiences as a therapeutic process. Psychological Science, 8, 162-166.

Pennebaker, J. W., Colder, M. \& Sharp, L. K. (1990). Accelerating the coping process. Journal of Personality and Social Psychology, 58, 528-537. 
Pennebaker, J. W., Hughes, C. F. \& O'Heeron, R. C. (1987). The psychophysiology of confession: Linking inhibitory and psychosomatic processes. Journal of Personality and Social Psychology, 52, 781-793.

Pennebaker, J. W. \& Beall, S. K (1986). Confronting a traumatic event: Toward an understanding of inhibition and disease. Journal of Abnormal Psychology, 95, 274-281.

Pennebaker, J. W. \& Francis, M. E. (1996). Cognitive, emotional, and language processes in disclosure. Cognition and Emotion, 10, 601-626.

Pennebaker, J. W., Kiecolt-Glaser, J. K. \& Glaser, R. (1988). Disclosure of traumas and immune function: Health implications for psychotherapy. Journal of Consulting and Clinical Psychology, 56, $239-245$.

Petrie, K. J., Fontanilla, I., Thomas, M. G., Booth, R. J. \& Pennebaker, J. W. (2004). Effect of written emotional expression on immune function in patients with human immunodeficiency virus infection: A randomized trial. Psychosomatic Medicine, 66, 272-275.

Petrie, K. J., Booth, R., Pennebaker, J. W., Davison, K. P. \& Thomas, M. (1995). Disclosure of trauma and immune response to hepatitis $B$ vaccination program. Journal of Consulting and Clinical Psychology, 63, 787-792.

Quintana, J. M., Padierna, A., Esteban, C., Aróstegui, I., Bilbao, A. \& Ruiz, I. (2003). Evaluation of the psychometric characteristics of Spanish version of the Hospital Anxiety and Depression scale. Acta Psychiatrica Scandinavica, 107, 216-221.

Sandín, B., Chorot, P., Lostao, L., Joiner, T., Santed, M. A. \& Valiente, R. M. (1999). Escalas PANAS de afecto positivo y negativo: validación factorial y convergencia transcultural. Psicothema, 11, 37-51.

Sloan, D. M. \& Marx, B. P. (2006). Exposure through written emotional disclosure: Two case examples. Cognitive and Behavioral Practice, 13, 227-234.

Sloan, D. M. \& Marx, B. P. (2004). A closer examination of the structured written disclosure procedure. Journal of Consulting and Clinical Psychology, 72, $165-175$.
Sloan, D. M., Marx, B. P. \& Epstein, E. M. (2005). Further examination of the exposure model underlying the efficacy of written emotional disclosure. Journal of Consulting and Clinical Psychology, 73, 549-554

Sloan, D. M., Marx, B. P., Epstein, E. M. \& Lexington, J. M. (2007). Does altering the writing instructions influence outcome associated with written disclosure? Behavior Therapy, 38, 155-168.

Smyth, J. M. (1998). Written emotion expression: Effect sizes, outcome types, and moderating variables. Journal of Consulting and Clinical Psychology, 66, 174-184.

Spera, S., Buhrfeind, E. \& Pennebaker, J. W. (1994). Expressive writing and job loss. Academy of Management Journal, 37, 722-733.

Stroebe, M., Schut, H. \& Stroebe, W. (2006). Who benefits from disclosure? Exploration of attachment style differences in the effects of expressing emotions. Clinical Psychology Review, 26, 66-85.

Stroebe, W., Schut, H. \& Stroebe, M. (2005). Grief work, disclosure and counseling: Do they help the bereaved? Clinical Psychology Review, 25, 395-414.

Tennant, C. (2006). Life events, stress and depression. Australian and New Zealand Journal of Psychiatry, 36, 173-182.

Warner, L. J., Lumley, M. A., Casey, R. J., Pierantoni, W., Salazar, R., Zoratti, E. M. et al. (2006). Health effects of written emotional disclosure in adolescents with asthma: A randomized, controlled trial. Journal of Pediatric Psychology, 31, 557-568.

Watson, D., Clark, L. A. \& Tellegen, A. (1988). Development and validation of brief measures of positive and negative affect: The PANAS scales. Journal of Personality and Social Psychology, 54, 1063-1070.

Wilson, K. G. \& Luciano, M. C. (2002). Terapia de aceptación y compromiso. Madrid: Pirámide.

Zigmond, A. S. \& Snaith, R. P. (1983). The Hospital Anxiety and Depression Scale. Acta Psychiatrica Scandinavica, 67, 361-370. 


\section{Anexo}

\section{Escala de síntomas físicos (W. Peñate)}

A continuación indica la frecuencia con la que te has sentido alguna molestia física durante aproximadamente el último mes, de acuerdo con las siguientes alternativas de respuesta:

$0=$ no ha ocurrido

$1=$ ha ocurrido de manera leve

$2=$ ha ocurrido de manera moderada

$3=$ ha ocurrido de manera grave

\begin{tabular}{|c|c|c|c|}
\hline Síntomas/molestias & 01 & 12 & 23 \\
\hline $\begin{array}{l}\text { Problemas de estomago (náuseas, vó- } \\
\text { mitos, diarreas...) }\end{array}$ & & & \\
\hline $\begin{array}{l}\text { Problemas de las vías respiratorias (tos, } \\
\text { catarro...) }\end{array}$ & & & \\
\hline Infecciones en la cavidad bucal & & & \\
\hline Infecciones en el aparato genital & & & \\
\hline Infecciones en la garganta & & & \\
\hline Infecciones cutáneas & & & \\
\hline $\begin{array}{l}\text { Alguna otra enfermedad vírica o bac- } \\
\text { teriana }\end{array}$ & & & \\
\hline
\end{tabular}

Ha visitado a algún centro de salud o algún profesional de la medicina ${ }^{4}$ en el último mes:

NO [ ] SI [ ], icuántas veces?........

4 No contabilizar las visitas por sufrir algún problema crónico o las que estaban programadas para llevar a cabo algún tipo de control o revisión. 